\title{
Fitness for work during the COVID-19 disease: principles and suggested assistive tool for protecting workers during the pandemic era
}

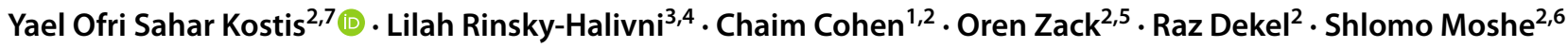

Received: 4 July 2020 / Accepted: 5 January 2021 / Published online: 1 June 2021

○ The Author(s), under exclusive licence to Springer-Verlag GmbH Germany, part of Springer Nature 2021

\begin{abstract}
Objective Amongst the many intricacies engendered by the COVID-19 pandemic was the posed risk of exposure to SARSCOV2 infections among workers and the need to evaluate fitness for work (FFW) of individuals in high exposure groups or those with background morbidity. A designated task force of the Israeli Association of Occupational Medicine has evaluated the relevant literature and published guidelines concerning medical work restrictions for employees in high-risk occupations during the epidemic of novel COVID-19.

Methods The study depicts a case series of 17 FFW evaluations performed in Maccabi Health Services occupational clinics using the guidelines formulated by the Israeli Occupational Medicine Association.

Results 17 patients, mainly healthcare workers, whose major health hazards were immunodeficiency, respiratory diseases and pregnancy, were assessed in this case series. We present the essential parameters of these FFW evaluations and discuss decision-making in selected cases.

Conclusions Applying guidelines in various work settings and health risk levels during the SARS-CoV-2 pandemic allows for both patients' health preservation and efficient workforce deployment.
\end{abstract}

Keywords Occupational exposure · Health risk assessment · Occupational groups (workers, healthcare workers) Occupational medicine (occupational health practice, exposure assessment, risk assessment) $\cdot$ Clinical guidelines

Yael Ofri Sahar Kostis

yael.sk@gmail.com; yaelsa3@ clalit.org.il

1 Department of Occupational Medicine, Tel Aviv-Yafo District, Clalit Health Services, Tel Aviv-Yafo, Israel

2 School of Public Health, Sackler Faculty of Medicine, Tel Aviv University, Tel Aviv-Yafo, Israel

3 Department of Occupational Medicine, Jerusalem District, Clalit Health Services, Jerusalem, Israel

4 Braun School of Public Health, Family Medicine Department, Hadassah-Hebrew University Medical School, Jerusalem, Israel

5 Department of Occupational Medicine, Israeli Defense Force, Ramat Gan, Israel

6 Department of Occupational Medicine, Maccabi Health Services, Center District, Tel Aviv-Yafo, Israel

7 Clalit Health Services, Occupational Department Tel Aviv-Yafo, 30 HaBosem St., Tel Aviv, Israel

$\begin{array}{ll}\text { Abbreviations } \\ \text { FFW } & \text { Fitness for work } \\ \text { MI } & \text { Myocardial infarction } \\ \text { RCOG } & \begin{array}{l}\text { Royal College of Obstetricians and } \\ \text { Gynaecologists }\end{array} \\ \text { ILO } & \text { International Labour Organization } \\ \text { IDSA } & \text { Infectious Diseases Society of America } \\ \text { IMA } & \text { Israeli Medical Association }\end{array}$

\section{Introduction}

Coronaviruses are significant human and animal pathogens. At the end of 2019, a novel coronavirus was first identified as the cause of a cluster of pneumonia cases in Wuhan, a city in the Hubei Province of China. COVID-19rapidly spread, resulting in an epidemic throughout countries throughout the world (McIntosh 2020).

Healthcare workers have been recognized as being at increased risk for developing infections presenting the community where their patients are drawn. These workers are 
often at the front line dealing with those who are ill and at the most infectious period of a disease, as in the cases of SARS, MERS, and Ebola (Baker et al. 2020). Other workers involved in providing public services may also be at increased risk during outbreaks where transmission occurs through close contact. A recent analysis in the USA has estimated that $10 \%$ of the workforce are employed in professions in which exposure to disease or infection occurs at least once per week (Semple and Cherrie 2020). Retail workers, bus drivers, cleaners, teachers, bank employees and hospitality staff are among the many service-sector employees who will have frequent and close interaction with many people over the course of a shift.

As the pandemic spread worldwide, millions of people were ordered to stay at home so as to minimize transmission of severe acute respiratory syndrome COVID-19, health-care workers were required to do the exact opposite. In April alone, there were an estimated 27 COVID-19-related health worker deaths in the USA, 106 in the UK, and 180 in Russia, with tens of thousands of infections (Lancet 2020). Healthcare workers account for up to $20 \%$ of all severely ill patients during the current pandemic (Assessment 2020).

Severe illness can occur in otherwise healthy individuals of any age, but it predominantly occurs in adults with advanced age or underlying medical comorbidities. The Center for Disease Control (CDC) website has published a list of disease and risk factors concerning COVID-19 (Centers for Disease Control and Prevention. People who are at higher risk for severe illness 2019 COVID-19, 2020). However, while such an approach, lacking specific consideration of disease clinical parameters and occupational exposure, was sufficient for the general public, health care workers and other workers under high exposure probability, diagnosed with a disease mentioned in that list, require a fitness for work (FFW) assessment, to decide whether or not they are fit for their work.

Fitness for work is defined as determining whether workers are fit to perform their duties with minimal risk to themselves or others. In Israel such evaluation is being taken by occupational health physician in a public service which is part of the National Health Service (Rinsky-Halivni 2020). The decision is often challenging and combines medical, ethical, economic, and legal considerations (Serra 2007). Clearly, a list of background medical conditions like diabetes, hypertension, ischemic heart disease, etc. is not precise enough to evaluate the workers' FFW in different occupations and settings. In the current scenario, different working sectors should be assured that all necessary preventive and protective measures are taken to minimize occupational safety and health risks. As general risk factors are neither precise nor practical enough in fitness for work settings, and no emphasis was given to potential occupational exposure to SARS-CoV-2 while defining at-risk populations, there was an obvious need for professional guidelines for the evaluation of workers' personal risk during the current pandemic.

A designated task force of the Israeli Association of Occupational Medicine has evaluated the relevant literature and published guidelines concerning medical work restrictions for employees in high-risk occupations during the epidemic of novel COVID-19 (Policy Paper 2020). This case series and review summarizes our recent experience in Israel in this subject of FFW in the COVID 19 epidemic utilizing the tool of the guidelines that weigh the aspects of work conditions and clinics that underpin the occupational health profession.

\section{Methods}

Data from 17 workers referred to Maccabi Health Services (MHS) occupational health clinics for FFW evaluation during March and April 2020 was collected. During that period of time, a total of 137 workers were assessed at the MHS occupational health clinics for various FFW etiologies. The study encompassed all workers referred for FFW evaluation due to potential occupational exposure to COVID-19.

Workers were referred for FFW evaluation as the COVID19 breakout raised questions regarding their capability of performing various works in various exposure settings, due to their medical condition and risk factors.

Demographic information including age and gender was collected, as well as detailed medical history and smoking history (represented by pack years). Medical history encompassed all electronic medical records available in medical registries, including specialty consulting medicine documentation (e.g. cardiology, rheumatology, etc.). Physical examinations were performed, and detailed job description were taken.

\section{All high-risk workers were evaluated using the Israeli Association of Occupational Medicine guidelines}

Major health hazard and the main occupational hazard for each patient were assessed. These two parameters were utilized to conclude FFW status. The severity of the relevant health hazard was classified by the Israeli Association of Occupational Medicine guidelines detailed in Appendix 1 (translated to English). Job categories were divided according to the level of exposure risk to COVID 19 into 4 categories: Very High Exposure Risk (1/4), High Exposure Risk (2/4), Medium Exposure Risk (3/4), Lower Exposure 
Risk (4/4). This exposure classification is integrated into the Israeli Occupational Medicine guidelines (Appendix 1) and was based on Occupational Safety and Health Administration's (OSHA) publications with the necessary adjustments for local settings (Guidance for occupational physicians providing advice on COVID-19 2020).

Very high exposure risk jobs (Category 1) are those with high potential for contact with known or suspected sources of COVID-19. For example, healthcare workers performing specific medical, postmortem, or laboratory aerosol-generating procedures on patients with high suspicion of being infected. High-risk exposure jobs (Category 2) include mainly healthcare personnel working for example in designated COVID 19 departments, but not those directly exposed to aerosols. Medium risk exposure jobs (Category 3 ) include those that require frequent and/or close contact with the general public. (e.g., school staff, high-population-density work environments, bus drivers, high-volume retail settings). In this specific exposure level, we recommend using screening and triage algorithms that included clinical symptoms inquiry (like whether costumers or patients are experiencing fever, cough, shortness of breath, sore throat, etc.) and temperature measurement (Wu 2019, Onder 2020). The use of such triage methods allows for costumers/patients reassignment according to the workers' personal health risk Lower exposure risk jobs (Category 4) are those that have minimal occupational contact with the public and can keep social distancing from other coworkers.

Job descriptions and occupational hazards were based on the International Hazard Datasheets on Occupations (HDO) published by the International Labour Organization (ILO) (International Hazard 2020), as well as the Dictionary Of Occupational Titles (DOT) published by the U.S. Dept. of Commerce, Bureau of the Census, USA government (Dictionary of Occupational Titles Index 2020).

The study used a convenience sample of consecutive cases, and, therefore, is not yet sufficient to validate the effectiveness of its application. In addition, the guidelines were applied to all high-risk workers, and no control group was assigned. The guidelines presented in this case series were only recently published when the study took place, and dealt with new, un-encountered occupational dilemmas. This case series is currently followed by a larger-scale study, hopefully to be published in the future.

Descriptive statistics were used to summarize the data; results were reported as ranges or means and standard deviations, as appropriate. Categorical variables were summarized as counts and percentages.

The case series was approved by Maccabi scientific institutional committee that dismissed the need for institutional ethics committees.

\section{Results}

All 17 patients included in the study were workers referred for FFW evaluation regarding their capability of performing various jobs in different exposure settings during COVID 19 epidemic. The demographic and clinical characteristics of the patients are shown in appendix 1 . The mean $( \pm$ SD) age of the patients was $47.7 \pm 12.7$ years (range, 31 to 68 ), $82 \%$ were women, $82 \%$ were non-smokers, and BMI was $24.8 \pm 3.9$. The major health hazards were, as expected, those which were reported as comorbidities endangering patients with COVID-19 (Wu 2019, Onder 2020, International Hazard 2020, Zhan 2020) such as immunocompromised patients $(6 / 17)$, cancer $(4 / 17)$, respiratory disease $(2 / 12)$, and pregnancy $(3 / 17)$.

The occupational characteristics of the patients are detailed in appendix 2. Most of the patients (14/17, 82\%) were healthcare workers employed at hospitals $(5 / 17,29 \%)$, community $(5 / 17,29 \%)$, and home care $(4 / 17,24 \%)$. Only 3 (18\%) were employed in administrative jobs. More than a third of the workers (6/17) were employed in very high/ high occupational exposure risk categories. The occupational health physician's decision was to restrict about half of the workers (9/17) from working in very high-risk or high-risk occupations. Recommendations for temporary re-deployment were given, respectively. No deviation from the guidelines' application were noted during the period of time evaluated by this study, but cases in which employers were thoroughly unable to implement the given occupational recommendations resulted in the temporary removal of the workers from their work stations. Clinical, laboratory, elaborated job descriptions and occupational hazards characteristics are detailed in Appendix 2.

We chose 4 cases and discussed them in detail, demonstrating the medical and occupational reasoning and decision making, based on the Israeli Occupational Medicine Association Guidelines. These cases are detailed in Appendix 3.

\section{Discussion}

The COVID 19 outbreak is an ongoing event. Governments worldwide struggle between the necessity to preserve the public's health and the maintenance of enduring economies. Nevertheless, it is necessary to maintain the health and vitality of the workforce. Occupational medicine takes pride in pursuing the perpetual quest to preserve workers' health and well-being, yet it constantly reflects over the hazards workers must sometimes confront as part of their occupation.

Healthcare workers have always had an increased risk of being infected by pathogens present in the community where their patients are drawn. Hunter et al. found $15-18 \%$ 
positive rate of SARS-CoV-2 tests among healthcare workers (Hunter et al. 2020). Several studies referred to this risk during the COVID-19 pandemic (Semple and Cherrie 2020, Lancet 2020, Assessment 2020).

Determining the acceptable extent of hazardous occupational exposure is not mere risk stratification of a work station, but an in-depth synthesis with the overall medical condition of the worker and its physiological, social and economic significance. Therefore, While formulating the guidelines for workers with possible occupational exposure to COVID-19, the task force consulted various specialists in the fields of medicine, occupational safety and health and human resources as well as professional medical committees to get consensus opinion, and reviewed the most updated evidence from the literature available for relevant mechanisms of damage, hazards control resources and specific relevance of chronic illnesses predisposing to severe illness due to COVID-19 illness.

The first version was issued on March 19th, 2020 and the following update on March 26th and May 6th, 2020.

When assessing FFW in workers with a potential exposure to SARS-CoV-2, the application of such principles is more challenging. Decision-making policies were based on risk stratification in two separate axes: the first is the degree of personal health risk resulting from the background illness and the second is the potential for occupational exposure to the virus.

In terms of increased health risk assessment, we set in the guidelines three major categories:

A. Medical conditions and treatments leading to immunodeficiency or immunosuppression-were a priori classified as being at increased risk. Oncology patients, those that underwent organ transplantation and patients with innate or acquired immune deficiencies are all defined as being at a higher risk for numerous infections in general (Fishman 2011; Ison and Hayden 2002; Meidani et al. 2014). A certain debate persists as to patients with decreased cytokine activity, who might have endure even a lighter course of COVID19 disease as they will lack the effect of the "cytokine storm". A potential role of anti-TNF agents as possible interventions to COVID-19 disease was also mentioned. The basic principle in managing immunosuppressed patients concludes that while they might suffer a less tumultuous course of disease, they are still more prone to severe morbidity and mortality due to infections, COVID-19 included. To define this group, we relied on the IDSA (Infectious Diseases Society of America) guidelines concerning immune deficient states and consulted immunologists on the need to emphasize definitions of humoral immunodeficiency.

B. Chronic medical conditions that could increase the risk for severe morbidity and mortality-When formulating this part, we partially relied on the CDC guidelines regarding high-risk chronic medical conditions-heart and lung diseases, hypertension, and diabetes. Lately, obesity (Simonnet 2020) and smoking have been added to the previous CDC risk factors, and these criteria had been included in the updated guidelines. When reviewing these risk factors, two parameters have been taken into consideration -

1. Disease severity-emphasis was given to moderate or higher degrees of each illness, either by accepted clinical definition of severity/severity scale, poor disease control or target organ impairments. We hypothesized that a more severe chronic disease would put patients at greater risk if infected with SARS-CoV-2, either because they had fewer baseline reserves or their disease course might be longer or more complicated due to specific impairments.

2. Disease complications directly compromising the immune system-relevant medical conditions such as recent myocardial infarction, diabetic ulcers or defects in the renin-angiotensin-aldosterone system were thus included, disregarding the severity of the underlying primary medical condition (Fang et al. 2015; Casqueiro et al. 2015; Singh et al. 2015).

C. Age- the CDC has defined age as an independent risk factor for COVID-19 morbidity and mortality (Groups at Higher Risk for Severe Illness 2020). The guidelines regarded workers aged 70 or older as being at increased risk, disregarding concomitant medical conditions. This cut-off point was based on the Israeli Ministry of Health recommendations. However, as many relevant chronic medical conditions are far more prevalent in populations aged 60 years of more (Atella et al. 2019; Adams 2017), and so occupational physician's assessment of such patients is advised.

Attention was also given to pregnant women-considering the short period of time in which SARS-CoV-2 has been identified and studied, there is an understandable difficulty in assessing the possible influences of the virus on pregnant women, fetuses, and neonates. Our guidelines relied on the Royal College of Obstetricians \& Gynaecologists (RCOG) pregnancy guidance for healthcare professionals, recently updated on May 22nd, 2020, which stated that there was no known evidence of increased morbidity in pregnant women, nor were there indications of significant disruption of the course of the pregnancy due to the virus, teratogenicity or miscarriages (COVID-19 virus infection and pregnancy 2020). As for women who became ill with COVID 19 during the later stages of their pregnancy, there is still the obstacle of distinguishing the general influences of severe morbidity (organ failure, need for ventilation, etc.) and the actual effects of COVID-19 on pregnancy outcomes (Schwartz 2020; Chen et al. 2020). It is advised to exclude pregnant workers from "very high" and "high" potential exposures, and to reassign them to lower potential exposure posts. 
As for the classification of potential occupational exposure to SARS-CoV-2, the occupational medicine guidelines definition for workplace risk levels below were based on the Occupational Risk Pyramid described in OSHA publication 3990 Guidance on Preparing Workplaces for COVID19 (Guidance for occupational physicians providing advice on COVID-19 2020) as mentioned above. "Very high" and "high" exposure risk levels are mainly compromised of healthcare providers, yet some of the exposures described in these levels can also exist in other occupational settings, mainly those requiring very close and prolonged personal contact (e.g. pre-school education staff, special-needs education staff nursing home caregivers, etc.). While emerging evidence point too many patients infected with SARS CoV2, especially young children, can be asymptomatic (Qiu et al. 2019), there is no comprehensive recommendation to include all the population as potential asymptomatic COVID 19 carriers. In some of the cases presented in the paper (cases numbers 12, 14, 16) due to the lack of available diagnostic tests and working in geographical regions that were mapped as highly afflicted with COVID 19, we rated some occupations as "very-high/high" exposure risk, especially if workers were required to perform aerosol inducing procedures.

When assessing each worker's FFW, a thorough job analysis should be performed. Every task was evaluated and the potential exposure risk to SARS-CoV-2 was determined based on the task placing the worker at the highest risk for exposure. In many cases, we aimed to maintain their work post by excluding workers from specific high-risk task/ tasks and redeploy to lower exposure levels, with suitable restrictions.

While most patients with the combination of both high personal risk and a very-high/high risk for occupational exposure to SARS CoV 2 should be either temporarily reassigned to lower exposure risk tasks or, as a last resort, temporarily excluded from their work, many of the patients categorized at a high personal risk but employed at medium exposure risk jobs can maintain their posts with the appropriate measures taken, as recommended by occupational medicine practitioner and the workplace guidance of the authorities.

As for patients working in low-exposure risk jobs, nearly all those who were fit for their work prior to the COVID 19 outbreak are capable of maintaining their work posts uninterrupted under the accepted precautions of social distancing, etc.

In conclusion, FFW assessment during a pandemic imposes a complicated yet feasible challenge, and requires the evaluation of specific work assignments and tasks and their respective potential exposure and the patient's health risk factors, as well as various protecting equipment and measures available and information about potential local outbreaks. The role of occupational medicine in such constellations is imperative. The incorporation and application of adequate occupational medicine guidelines can assist present and future policy making, aid occupational health physicians, employers and employees, ensuring both workers' health protection and a viable workforce.

Supplementary Information The online version contains supplementary material available at https://doi.org/10.1007/s00420-021-01722-x.

Funding None.

Availability of data and materials The datasets generated and/or analyzed during the current study are not publicly available due to their clinical and personal nature. Study datasets are documented within patient medical records. Due to their personal nature, they are not readily available but are available from the corresponding author on reasonable request.

\section{Declarations}

Ethics approval and consent to participate The study was approved by the scientific institutional committee that dismissed the need for institutional ethics committees.

Consent for publication Not applicable.

Competing interests The authors declare that they have no competing interests.

\section{References}

Adams ML (2017) Differences between younger and older us adults with multiple chronic conditions. CDC, Prev Chronic Dis 14:160613

Assessment RR. Coronavirus disease 2019 (COVID-19) in the EU/ EEA and the UK - eighth update. https://www.ecdc.europa.eu/ sites/default/files/documents/covid-19-rapid-risk-assessmentcoronavirus-disease-2019-eighth-update-8-april-2020.pdf. Accessed 8 Apr 2020

Atella V, Piano Mortari A, Kopinska J, Belotti F, Lapi F, Cricelli C, Fontana L (2019) Trends in age-related disease burden and healthcare utilization. Aging Cell 18(1):e12861

Baker MG, Peckham TK, Seixas NS (2020) Estimating the burden of United States workers exposed to infection or disease: a key factor in containing risk of COVID-19 infection. PLoS ONE. https://doi. org/10.1101/2020.03.02.20030288v1.full.pdf

COVID-19 virus infection and pregnancy, Occupational health advice for employers and pregnant women during the COVID19 pandemic,Version 3: Published Tuesday 21 April 2020, Royal College of Obstetricians and Gynaecologists. https://www.rcog. org.uk/globalassets/documents/guidelines/2020-04-21-occup ational-health-advice-for-employers-and-pregnant-women.pdf. Accessed 20 May 2020

Casqueiro J, Casqueiro J, Alves C (2012) Infections in patients with diabetes mellitus: A review of pathogenesis. Indian J Endocrinol Metab 16(Suppl1):S27-S36

Centers for Disease Control and Prevention. People who are at higher risk for severe illness 2019 COVID-19. https://www.cdc.gov/coron avirus/2019-ncov/need-extra-precautions/people-at-higher-risk. 
html?CDC_AA_refVal=https $\% 3 \mathrm{~A} \% 2 \mathrm{~F} \% 2 \mathrm{Fwww} . c d c . g o v \% 2 \mathrm{Fcor}$ onavirus\%2F2019-ncov\%2Fspecific-groups\%2Fhigh-risk-compl ications.html. Accessed 20 May 2020

Chen H, Guo J, Wang C, Luo F, Yu X, Zhang W, Li J, Zhao D, Xu D, Gong Q, Liao J, Yang H, Hou W, Zhang Y (2020) Clinical characteristics and intrauterine vertical transmission potential of COVID-19 infection in nine pregnant women: a retrospective review of medical records. Lancet 395:809-815. https://doi.org/ 10.1016/S0140-6736(20)30360-3

Dictionary of Occupational Titles Index. https://occupationalinfo.org/ dot_index.html\#MENU. Accessed 20 May 2020

Fang L, Moore XL, Dart AM, Wang LM (2015) Systemic inflammatory response following acute myocardial infarction. J Geriatr Cardiol 12(3):305-312

Fishman JA (2011) Infections in immunocompromised hosts and organ transplant recipients: Essentials. Liver Transpl 17(Suppl 3):S34S37. https://doi.org/10.1002/1t.22378

Groups at Higher Risk for Severe Illness, Centers for Disease Control and Prevention. https://www.cdc.gov/coronavirus/2019-ncov/ need-extra-precautions/groups-at-higher-risk.html. Accessed 20 May 2020

Guidance for occupational physicians providing advice on COVID-19. https://www.osha.gov/Publications/OSHA3990.pdf?deliveryNa me=USCDC-10_4-DM23416\&deliveryName=FCP_2_USCDC10_4-DM23416

Hunter E, Price DA, Elizabeth Murphy E et al (2020) First experience of COVID-19 screening of health-care workers in England. Lancet 2(395): 10234

International Hazard Datasheets on Occupations, International Labour organization. https://www.ilo.org/safework/info/publications/ WCMS_113135/lang--en/index.htm. Accessed 20 May 2020

Ison MG, Hayden FG (2002) Viral infections in immunocompromised patients: what's new with respiratory viruses? Curr Opin Infect Dis 15(4):355-367

Lancet T (2020) The plight of essential workers during the COVID-19 pandemic Editorial. Lancet 395:1587

McIntosh K, Hirsch MS, Bloom A (2019) Coronavirus disease 2019 (COVID-19). https://www-uptodate-com.rproxy.tau.ac.il/conte nts/coronavirus-disease-2019-covid-19-epidemiology-virologyclinical-features-diagnosis-and-prevention?search=covid $\% 2019 \&$ source $=$ search_result\&selectedTitle $=1 \sim 150 \&$ usage_type $=$ defau lt\&display_rank $=1$. Accessed 22 May 2020

Meidani M, Emami Naeini A, Rostami M, Sherkat R, Tayeri K (2014) Immunocompromised patients: review of the most common infections happened in 446 hospitalized patients. J Res Med Sci 19(Suppl 1):S71-S73
Onder G, Rezza G, Brusaferro S (2020) Case-fatality rate and characteristics of patients dying in relation to COVID-19 in Italy. JAMA 323(18):1775-1776. https://doi.org/10.1001/jama.2020.4683

Policy Paper: Evaluation of Work Capacity for Workers of Different Exposure Levels to the New Corona Virus (SARS CoV 2), in light of the COVID 19 pandemic, Israeli Association of Occupational Medicine. https://www.ima.org.il/userfiles/image/refuahTaasukati tCorona032020.pdf. Accessed 6 Apr 2020

Qiu H, Wu J, Hong L, Luo Y, Song Q, Chen D (2019) Clinical and epidemiological features of 36 children with coronavirus disease 2019 (COVID-19) in Zhejiang, China: an observational cohort study. Lancet Infect Dis. https://doi.org/10.1016/S1473-3099(20) 30198-5

Rinsky-Halivni L, Cohen C, Moshe S, Amster E (2020) Socialized occupational medicine in Israel: past, present, and future. Arch Environ Occup Health 75(1):45-55

Schwartz DA (2020) An Analysis of 38 Pregnant Women with COVID19 , their newborn infants, and maternal-fetal transmission of SARS-CoV-2: maternal coronavirus infections and pregnancy outcomes. Arch Pathol Lab Med. https://doi.org/10.5858/arpa. 2020-0901-SA

Semple S, Cherrie JW (2020) Covid-19: Protecting Worker Health. Ann Work Expos Health 1:1-4

Serra C, Rodriguez MC, Delclos GL, Plana M, Gómez López LI, Benavides FG (2007) Criteria and methods used for the assessment of fitness for work: a systematic review. Occup Environ Med 64(5):304-312

Simonnet A, Chetboun M, Poissy J, Raverdy V, Noulette J, Duhamel A, Labreuche J, Mathieu D, Pattou F, Jourdain M (2020) High prevalence of obesity in severe acute respiratory syndrome coronavirus-2 (SARS-CoV-2) requiring invasive mechanical ventilation. Obesity

Singh MV, Chapleau MW, Harwani SC, Abboud FM (2014) The immune system and hypertension. Immunol Res 59:243-253

Wu C, Chen X, Cai Y, Zhou X, Xu S, Huang H, Zhang L, Zhou X, Du C, Zhang Y, Song J (2019) Risk factors associated with acute respiratory distress syndrome and death in patients with coronavirus disease 2019 pneumonia in Wuhan China. JAMA Intern Med 180(7):934-943

Zhan M, Qin Y, Xue X, Zhu S (2020) Death from Covid-19 of 23 Health Care Workers in China. N Engl J Med 382(23):2267-2268

Publisher's Note Springer Nature remains neutral with regard to jurisdictional claims in published maps and institutional affiliations. 